\section{Life is fragile: FMRP controls cell death in liver disease}

\author{
Mihael Vucur, Tom Luedde 두
}

Acute and chronic liver diseases due to various pathogens have in common that cellular stress and cell death represent their initial triggers. While in acute liver damage and especially in acute liver failure, the loss of functional hepatocytes is the dominant, life-limiting factor, low levels of cell death in chronic diseases like non-alcoholic fatty liver (NASH) or alcoholic liver disease initiate a cascade of immunological cell death responses, driving the progression to liver cirrhosis and finally to hepatocellular carcinoma. ${ }^{1}$ On a molecular level, hepatocytes that undergo programmed cell death release factors known as danger-associated molecular patterns, which activate immune cells (macrophages) and hepatic stellate cells, and are thus involved in chronic phase transition of liver diseases and decompensation. $^{1}$

For years, the term apoptosis was used synonymously with the term 'programmed cell death'. Nevertheless, this highly simplified dogma was swept away over night when it was discovered that apoptosis was accompanied by several other programmed cell death signalling pathways, and that necrosis was not only a passive form of cell death, for example, in

Division of Gastroenterology, Hepatology and GI Oncology, University Hospital RWTH Aachen, Aachen, Germany

Correspondence to Dr Tom Luedde, Division of Gastroenterology, Hepatology and GI Oncology, University Hospital RWTH Aachen, Aachen 52074, Germany; tluedde@ukaachen.de the context of ischaemia, but that necrosis, could also be regulated. ${ }^{2}$ One important form of programmed necrosis is called necroptosis mediated by the molecules receptor-interacting serine/threonineprotein kinase (RIPK)-1, RIPK3 and mixed lineage kinase domain-like protein (MLKL). ${ }^{3}$ The exact role of necroptosis in liver biology and pathophysiology is currently not well understood, which is mainly due to the lack of simple and specific tools to detect activation of necroptosis in vivo. ${ }^{3}$ However, it was shown that necroptosis is the dominant cell death pathway over apoptosis in NASH and NASH fibrosis. ${ }^{4}$

At present, it is not fully understood how necroptosis works, but it is assumed that by different phosphorylation and ubiquitination processes, for example, by tumour necrosis factor (TNF) stimulation and simultaneous inhibition of caspase- 8 , the formation of a RIPK1-RIPK3-containing complex, the so-called necrosome, leads to activation and multimerisation of MLKL molecules that form pores within the cell membrane and execute necrosis. ${ }^{5}$ Interestingly, new interactions of molecular signalling pathways have recently been discovered that previously had not been associated with the execution of apoptosis or necroptosis. ${ }^{6} 7$ Therefore, the molecular regulation of the apoptosis and necroptosis machinery might be more complex than previously suspected, suggesting that further molecular interactions with these pathways might soon be discovered.
In the paper of Zhuang and colleagues published in $G u t,{ }^{8}$ the authors provide evidence for a previously unrecognised role of fragile $\mathrm{X}$ mental retardation protein (FMRP) in protecting the liver from TNF-induced cell death. Mutations in the FMR1-gene encoding for FMRP represent a major cause of inherited intellectual disability within the fragile $\mathrm{X}$ mental retardation (FMR) syndrome, but the role of this gene outside the central nervous system and specifically in the liver was previously unclear. In their paper, they provide evidence that FMRP has a protective role in several liver injury models including cholestasis, septic shock models and viral hepatitis (figure 1), which might have important clinical implications not only for FMR patients but also for the development of novel protection strategies in acute and chronic liver disease.

On a molecular level, the authors provide evidence that FMRP might control the activation of RIPK1, a central signalling nexus involved in the regulation of both apoptosis and necroptosis in the liver. ${ }^{10-12}$ In this line, the authors provided quite intriguing data suggesting the activation of both of these cell death pathways in FMRP-defective liver cells (figure 1). ${ }^{8}$ On one hand, these data are in line with the dual role of RIPK1 in controlling apoptosis and necroptosis. ${ }^{12}$ On the other hand, there are not many examples that we know of that clearly showed that cells do activate both cell death pathways simultaneously. Therefore, it would be of high interest to clarify the molecular role of FMRP on a deeper molecular level and specifically nail down the exact molecular interaction between FMRP and RIPK1 and/or other molecules of the apoptosis and necroptosis machinery. Ultimately, this interesting current study and future studies on FMRP and other comodulatory 


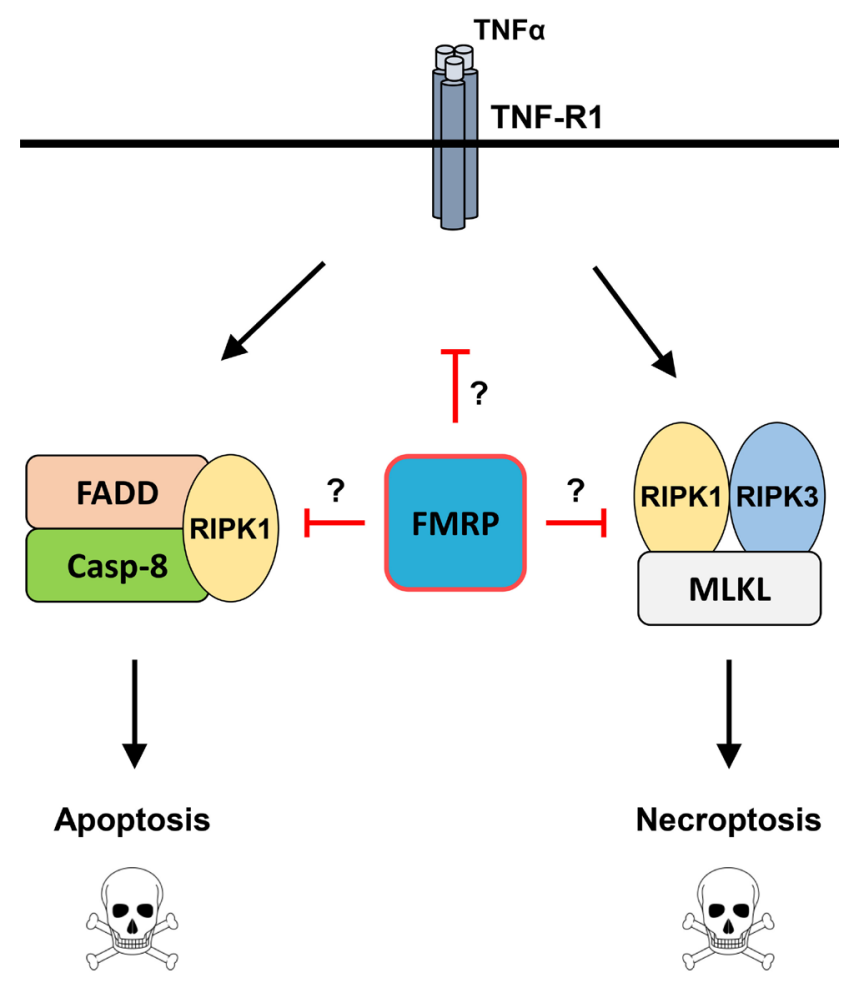

Figure 1 The deficiency of FMRP triggers context-specific, TNF-mediated apoptosis and necroptosis in liver tissue. In FMRP-deficient mice, liver injury following bile duct ligation or TNF/ GalN administration can be alleviated by Nec-1s treatment, indicating RIPK1 dependent cell death activation. FMRP's direct target(s) still need(s) to be explored. FADD, FAS-associated death domain protein; FMRP, fragile X mental retardation protein; MLKL, mixed lineage kinase domain-like protein; RIPK,receptor-interacting serine/threonine-protein kinase; TNF, tumour necrosis factor.

molecules might reveal that our unidimensional, linear view of cell death signalling pathways is too simplistic, and that instead transitional states between cell death signalling pathways exist that will make it much more complex to define specific clinical targeting strategies.

Contributors MV and TL wrote the manuscript.

Funding TL was funded by an ERC Consolidator grant (771083-Phase Control), by the German Cancer Aid (Deutsche Krebshilfe 110043 and a Mildred-ScheelProfessorship), the German-Research Foundation (SFB CRC, project A01, SFB-TRR57/P06 and LU 1360/3-1), the Interdisciplinary Centre for Clinical Research within the Faculty of Medicine at the RWTH Aachen University
(IZKF) and a grant from the medical faculty of the RWTH Aachen. MV was funded by the Interdisciplinary Centre for Clinical Research within the Faculty of Medicine at the RWTH Aachen University (IZKF Aachen, Project 03-8) and a Grant from the medical faculty of

Competing interests None declared.

Patient consent for publication Not required.

Provenance and peer review Commissioned; internally peer reviewed.

(C) Author(s) (or their employer(s)) 2020. No commercial re-use. See rights and permissions. Published by BMJ.

To cite Vucur M, Luedde T. Gut 2020;69:2-3. the RWTH Aachen (START).

\section{Check for updates}

Received 4 September 2019

Revised 17 September 2019

Accepted 18 September 2019

Published Online First 25 September 2019

\section{S Linked}

- http://dx.doi.org/10.1136/gutjnl-2019-318215

Gut 2020;69:2-3.

doi:10.1136/gutjnl-2019-319534

\section{ORCID iD}

Tom Luedde http://orcid.org/0000-0002-6288-8821

\section{REFERENCES}

1 Luedde T, Kaplowitz N, Schwabe RF. Cell death and cell death responses in liver disease: mechanisms and clinical relevance. Gastroenterology 2014;147:765-83.

2 Galluzzi L, Vitale I, Aaronson SA, et al. Molecular mechanisms of cell death: recommendations of the nomenclature Committee on cell death 2018. Cell Death Differ 2018:25:486-541.

3 Schwabe RF, Luedde T. Apoptosis and necroptosis in the liver: a matter of life and death. Nat Rev Gastroenterol Hepatol 2018;15:738-52.

4 Gautheron J, Vucur M, Reisinger F, et al. A positive feedback loop between RIP3 and JNK controls non-alcoholic steatohepatitis. EMBO Mol Med 2014:6:1062-74.

5 Weinlich R, Oberst A, Beere HM, et al. Necroptosis in development, inflammation and disease. Nat Rev Mol Cell Biol 2017;18:127-36.

6 McNamara DE, Dovey CM, Hale AT, et al. Direct activation of human MLKL by a select repertoire of inositol phosphate metabolites. Cell Chem Biol 2019:26:863-77.

7 Najafov A, Mookhtiar AK, Luu HS, et al. TAM kinases promote necroptosis by regulating oligomerization of MLKL. Mol Cell 2019;75:457-68.

8 Zhuang $Y$, Xu HC, Shinde PV, et al. Fragile X mental retardation protein protects against tumour necrosis factor-mediated cell death and liver injury. Gut 2019;69:133-45

9 Bagni C, Tassone F, Neri G, et al. Fragile X syndrome: causes, diagnosis, mechanisms, and therapeutics. J Clin Invest 2012:122:4314-22.

10 Schneider AT, Gautheron J, Feoktistova M, et al. RIPK1 suppresses a TRAF2-dependent pathway to liver cancer. Cancer Cell 2017:31:94-109.

11 Schneider AT, Gautheron J, Tacke F, et al. Receptor interacting protein kinase 1 (RIPK1) in hepatocytes does not mediate murine acetaminophen toxicity. Hepatology 2016:64:306-8.

12 Vucur M, Schneider AT, Gautheron J, et al. The enigma of RIPK1 in the liver: more than just a kinase. Mol Cell Oncol 2017;4:e1304191. 